\title{
Frequency Domain Analyses of Neonatal Flash VEP
}

\author{
JOSEPH R. ISLER, JILLIAN GROSE-FIFER, WILLIAM P. FIFER, SARAH HOUSMAN, RAYMOND I. STARK, \\ AND PHILIP G. GRIEVE
}

\author{
Department of Pediatrics [J.R.I., J.G.-F., R.I.S., P.G.G.], Department of Psychiatry [W.P.F., S.H.], Columbia College of Physicians and \\ Surgeons, New York, New York 10032
}

\begin{abstract}
A battery of frequency-dependent measures was made for high-density electroencephalographic recording measured in response to a flash stimulus in 12 normal term infants within $2 \mathrm{~d}$ of birth. Significant changes recorded in posterior electrodes in the same time window as the visual evoked potential (VEP) included increased local synchrony at $\sim 40 \mathrm{~Hz}$, increased power at $\sim 16 \mathrm{~Hz}$, the emergence of nonlinear coupling of lower $(\sim 2 \mathrm{~Hz})$ and higher frequency oscillations, and phase locking over a wide range of frequencies. This research provides evidence of neural synchrony and nonlinear coupling in response to a simple visual stimulus. These mechanisms are functional in early development. It is proposed that the frequency domain metrics of this study may augment current diagnostic measures of cortical function at birth. (Pediatr Res 62: 581-585, 2007)
\end{abstract}

$\mathrm{T}$ The flash visual evoked potential (VEP) has been widely used during infancy for tracking developmental changes in visual maturation and assessing cortical function at birth; yet its clinical utility in the assessment of cortical function has been debated (1-8). The diagnostic inadequacy of traditional (time domain) VEP analysis in early central nervous system assessment motivated the present study, which investigates how a battery of frequency domain analyses of VEP data can be used as alternative and complementary measures of the neural dynamics generating the response. The goal of the work was to quantify the cortical response to a flash stimulus with measures directly related to known neural mechanisms. Ultimately, this approach could offer a more comprehensive clinical assessment of early cortical development (9).

Event-related potentials (ERPs), such as the VEP, result from averaging the electroencephalographic (EEG) activity across many repetitions of a stimulus. ERPs represent the time-locked average response. Averaging serves to minimize the amplitude of any randomly occurring spontaneous EEG activity in the ERP. The extent to which ongoing EEG activity participates in the ERP is an important issue. At one extreme, spontaneous EEG activity can be interpreted as additive noise to an invariant waveform in which case averaging is justifiable because it serves to remove the noise. Alternatively, the ERP could be due to the stimulus resetting the phase of spontaneous EEG activity. In reality, both processes could occur to some extent. Regardless of which process predominates, the

Received January 12, 2007; accepted June 13, 2007.

Correspondence: Joseph R. Isler, Ph.D., Department of Pediatrics, Columbia College of Physicians and Surgeons, 630 West 168th Street, New York, NY 10032; e-mail: jri2101@ columbia.edu

NIH grants 5K25NS052230 and HD032774.
EEG will show a nonrandom phase at the time of a prominent ERP peak or trough, and so a frequency domain measure of phase locking provides an alternative way to detect ERP components (10). Other frequency-dependent measures characterize aspects of the cortical response that are difficult or impossible to assess with the ERP alone. For example, synchronization and/or coupling of brain activity across different cortical areas can be assessed using measurements of highfrequency synchrony or cross-frequency coupling, with no requirement that such activity be time-locked to the stimulus.

The nature of the spontaneous EEG/ERP relationship relates to the more fundamental question regarding the role of local field potentials in information encoding and signaling within neural networks. It is increasingly apparent that local field potentials and the EEG oscillations that they generate are not mere epiphenomena or noise, but instead play a key role in the ways in which the brain processes and integrates information $(11,12)$. Synchrony of neuronal oscillations provides an efficient mechanism for controlling the gating and gain of information flow (12) and has been shown to correlate with memory consolidation (13). Synchronization of oscillations within and between cortical areas appears to be a fundamental mechanism in both perception $(14,15)$ and behavior control $(16,17)$. Synchronization at relatively high frequencies (30-50 $\mathrm{Hz}$ or gamma band) predominates within local networks (18) and is thought to effect feature binding in visual cortex $(19,20)$. Furthermore, there is evidence of distinct functional roles of oscillations in different frequency bands (13). Nonlinear phase coupling between frequencies allows local networks to participate in more distributed assemblies (21-23). Such coupling provides a mechanism for connecting local and global network dynamics: information encoded locally in the phase of a high-frequency oscillation can be preserved across cycles of a lower frequency oscillation that serves to unite the neural assembly globally.

Most EEG power resides at the lowest frequencies, so not surprisingly ERP waveforms are dominated by peaks and troughs with constituent frequencies of $\sim 10 \mathrm{~Hz}$ or less. Therefore, traditional time domain-averaged waveforms are insensitive to activity in the gamma band. Conversely, frequency domain methods that explicitly measure synchrony within and between brain regions (coherence, phase synchrony) or nonlinear cross-frequency coupling (bicoherence)

Abbreviations: ERP, event-related potential; FFT, fast Fourier transform; VEP, visual evoked potential 
can provide more sensitive measures of even low amplitude oscillations over a broad frequency range. Moreover, such measures quantify specific mechanisms of neural network dynamics. With these considerations in mind, we hypothesized visual stimulation would evoke a robust increase in synchrony in the gamma band over occipital cortex, indicating that local neurons in the visual cortex are responding concurrently. In addition, we expected that there would be evidence of nonlinear phase coupling between lower and higher frequencies in the same region, indicating that local activity related to the stimulus may be coupled to activity in other brain regions. Validation of these hypotheses would suggest new measures for the diagnosis of cortical integrity in infancy.

\section{METHODS}

Subjects. This study was approved by the Institutional Review Boards at the New York State Psychiatric Institute and Columbia University. The subjects were infants within $2 \mathrm{~d}$ of birth in the Well Baby Nursery of the Morgan Stanley Children's Hospital of New York Presbyterian and Columbia University Medical Center. Each infant's mother gave her informed consent to the study. Data were collected from 19 neonates (five female, 14 male).

Protocol, measurements, and data preprocessing. Multiple (four to eight) blocks of thirty 100-ms white light flashes were delivered to the neonates at a rate of one flash per second using a Grass PS33 flash unit (at intensity level 8) held approximately $20 \mathrm{~cm}$ from the eyes. Electrocardiographic activity and respiration, sampled at 500 and $20 \mathrm{~Hz}$, respectively, were recorded for scoring sleep state. Electroencephalographic data were simultaneously recorded using an Electrical Geodesics Inc. system with a 128-electrode net fitted over the infant head (ocular electrodes normally affixed to the face were not used in this study). Neighboring electrodes in the net were separated by $\sim 1 \mathrm{~cm}$. Data were sampled at $1000 \mathrm{~Hz}$ and hardware filtered below $400 \mathrm{~Hz}$ and above 0.1 $\mathrm{Hz}$. After data recording, $60 \mathrm{~Hz}$ and its harmonics up to $360 \mathrm{~Hz}$ were removed with a series of 4-Hz wide 1600-point linear phase notch filers with $60 \mathrm{~dB}$ falloff at $2 \mathrm{~Hz}$ from the notch. All analyses were performed using Matlab programming.

Trials with eye movements were rejected from further processing. Eye movements were detected automatically using a bipolar montage of smoothed (40-point boxcar) raw data from 11 channels near the eyes. To eliminate bad electrodes triggering eye movement detection, bipolar channels with rms amplitude $>40 \mathrm{mV}$ were removed from the montage. Trials were rejected if four or more of the remaining bipolar channels had an absolute first derivative $>0.5 \mathrm{mV}$ per sample. Visual inspection of remaining trials removed trials with eye movements not detected automatically. Head movement and other artifact were detected for each trial, and channels were rejected if any of the following criteria were met: absolute sample-to-sample change $>25 \mathrm{mV}$, absolute value $>50 \mathrm{mV}$, spectral slope between 20 and $200 \mathrm{~Hz}$ greater than -0.1 . Trials with $>16 \%$ of channels matching one of the above criteria were rejected. Finally, data were re-referenced to the average reference of artifactfree channels for all subsequent analyses. Infants with fewer than 25 trials remaining were excluded, leaving a total 12 infants (10 male, two female) in the study group. The mean number of trials across infants was 55 .

Data analysis. Averaged waveforms were computed as the mean voltage over time across trials at each electrode with the removal of the mean voltage from a prestimulus $128 \mathrm{~ms}$. For the computation of frequency domain quantities, each trial was divided into eight nonoverlapping 128-ms segments (resolution $\sim 7.8 \mathrm{~Hz}$ ) with one prestimulus segment and seven poststimulus segments. Fast Fourier transforms (FFTs) were taken for each segment after removal of the segment mean (de-meaning) and application of a Hann window. De-meaning shifts the center of the lowest frequency bin $(-4$ to $4 \mathrm{~Hz}$ ) from 0 to $2 \mathrm{~Hz}$. Power spectra were the magnitude-squared FFTs averaged across trials. Cross-spectra were calculated by averaging the product of one channel FFT with the complex conjugate FFT of another channel for all possible pairs of channels across trials. Coherence was defined as the magnitude of the cross spectrum normalized by channel power (24). Phase synchrony between pairs of electrodes was measured as 1 minus the circular variance across trials of the difference between FFT phases. Local coherence (local phase synchrony, local cross-spectrum) was taken as the mean coherence (phase synchrony, cross-spectrum) between an electrode and its nearest neighbors. Phase-locking at a single electrode was measured as 1 minus the circular variance across trials of the FFT phase. Bicoherence at a single electrode was measured using the product of the FFT at two different frequencies times the complex conjugate of the FFT at the sum of the two frequencies, normalized by power at the respective frequencies (25).

Changes from prestimulus (baseline) values were computed for each frequency domain quantity. Most measures (with the exception of bicoherence and phase-locking) had large nonzero baseline values and so were described in terms of fractional change from baseline. To correct for spatial errors due to variations in electrode net placement, cortical anatomy and highly variable neonatal head shapes, before group averaging all quantities for each infant, were spatially smoothed at each electrode with a spatial boxcar window including its nearest-neighbor electrodes. Subsequently, group statistics (mean, SE, significance levels) were calculated for all change scores. A region of interest over the visual cortex was defined using a set of 15 posterior electrodes closely positioned over the occipital and posterior parietal lobes. For each frequency domain measurement, a rough estimate of sensitivity to the neural dynamics generating the VEP (hereafter called VEP sensitivity estimate) was obtained by averaging the corresponding change score over the 15 electrodes in the region of interest (over all pairs in the region for bivariate quantities) and then plotting the grand mean and SE as a function of time. For all other results, a $t$ test of change from baseline was used to determine $p$ values for each electrode or pair of electrodes. A false discovery rate (26) of 0.05 was used to correct for multiple comparisons across all electrodes (or across all pairs for bivariate quantities), except for local phase synchrony (see Fig. 4), which was made using a false discovery rate of 0.07 .

\section{RESULTS}

Averaged waveforms. For the 12 infants included in the study, a positive peak at $\sim 200$-ms latency was clearly present in six subjects, whereas two infants showed no VEP, and for the rest it was difficult to discern or ambiguous. Sleep state did not appear to systematically affect the waveform; however, there were not enough babies in both sleep states to quantify any sleep-dependent differences between states. The VEP had variable temporal morphology across infants, with broader peaks in some infants than others, and the presence/absence of other components was variable as well. In four cases, VEP amplitudes were greater at left posterior electrodes than at right posterior electrodes and in all cases were greater over posterior regions than central and frontal regions. The group mean VEP, shown in Figure 1, was not statistically significant at any electrode, i.e. the grand average waveform from 100 to $300 \mathrm{~ms}$ post-stimulus was not significantly different from zero at a false discovery rate of 0.05 .

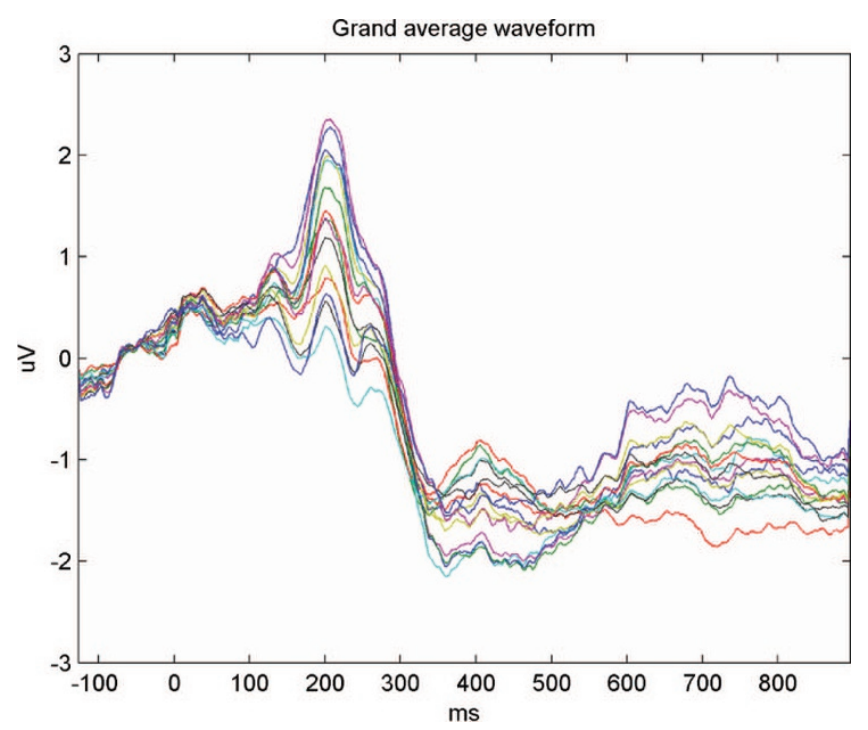

Figure 1. Overlaid plots of grand averaged $(n=12)$ waveforms at 17 posterior electrodes. 
Comparison of frequency domain measures. A comparison of VEP sensitivity estimates of the frequency domain measures across neonates is shown in Figure 2. Band power, cross-spectrum (not shown but very similar to band power), phase-locking, and bicoherence showed significant changes occurring in the same time window as the VEP, thus demonstrating the sensitivity of these measures to the neural dynamics underlying the VEP. Sensitivity was generally greatest at lower frequencies, although small but significant changes were also apparent at higher frequencies, including the gamma band. Coherence and phase synchrony VEP sensitivity estimates are not shown because they exhibited very small $(<10 \%)$ nonsignificant changes.

Power spectra and local cross-spectra. Figure 3 shows the fractional change in band power at $16 \mathrm{~Hz}$. In this and subsequent figures, only the first four post-stimulus times are included (subsequent times were unremarkable). Significant band power changes are exclusively positive and occur primarily in the same time window as the VEP. Power changes of $100 \%$ (indicated by the red/brown circles) occur over posterior regions in the $16-\mathrm{Hz}$ band. Smaller significant band power effects occur in frontal and central regions and at lower frequencies (not shown). Changes in the locally averaged cross-spectra are significant at a few more electrodes than band power, but exhibit the same spatial distribution. Even the

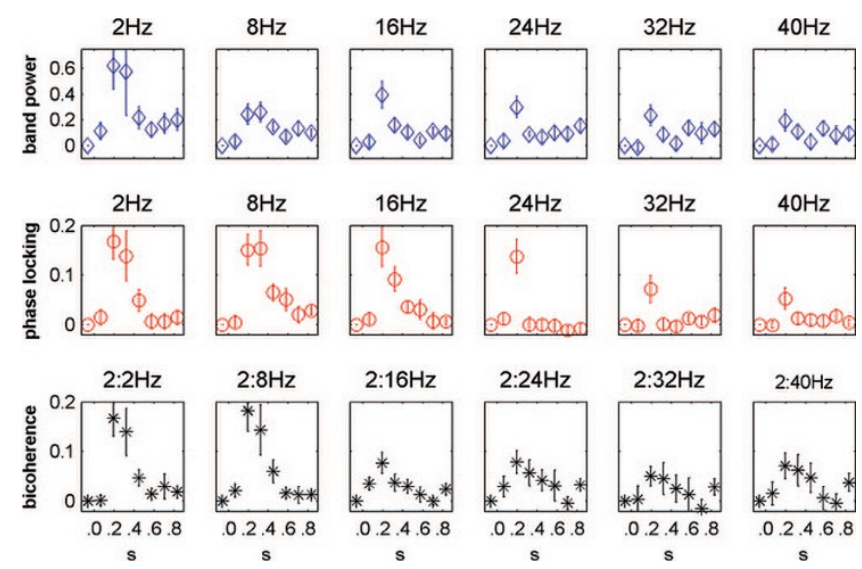

Figure 2. Comparison of VEP sensitivity estimates showing fractional change in band power (top row), change in phase-locking (middle row), and change in bicoherence (bottom row). Values are group means and SEs of quantities averaged over posterior electrodes. Times are fractional seconds post-stimulus.

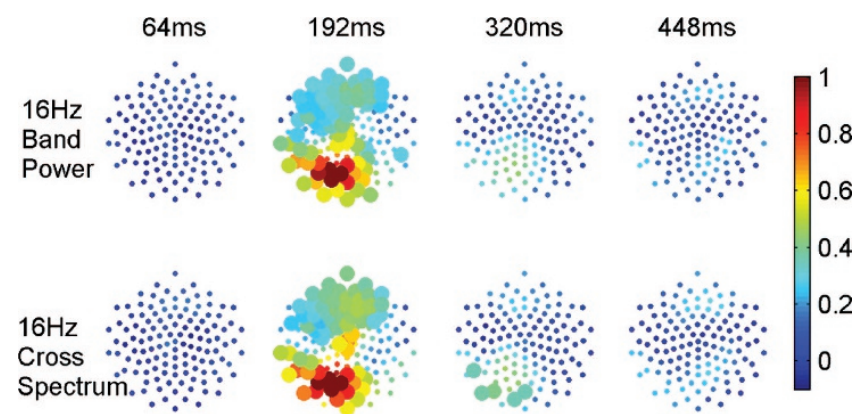

Figure 3. Band power and local cross-spectrum fractional change at $16 \mathrm{~Hz}$. Significant results (false discovery rate $=0.05$ ) are shown with large circles; times are post-stimulus milliseconds. nonsignificant results show spatial clustering over left posterior regions.

Synchrony. For all 7550 possible pairs of electrodes, coherence and phase synchrony were evaluated at frequencies from 2 to $40 \mathrm{~Hz}$. For all 125 electrodes, local measures of coherence and phase synchrony were evaluated by averaging over nearest-neighbor pairs. The only synchrony measure that was significant was local phase synchrony at $40 \mathrm{~Hz}$ in two clusters of left posterior and right anterior electrodes (Fig. 4).

Phase-locking. Phase-locking, which measures phase nonrandomness, showed significant increases of $\sim 0.3$ over left/ central posterior regions at frequencies up to $24 \mathrm{~Hz}$. Smaller significant changes occurred over frontal regions across all frequencies (Fig. 5).

Bicoherence. The bicoherence response for phase couplings between $2 \mathrm{~Hz}$ and all other frequencies (from 2 to 24 $\mathrm{Hz}$ ) are shown in Figure 6. Significant increases $>0.15$, occurred at occipital electrodes in the VEP time window for 2 $\mathrm{Hz}: 2 \mathrm{~Hz}$ coupling and for $2 \mathrm{~Hz}: 8 \mathrm{~Hz}$ coupling. Smaller significant changes were found over frontal areas. However, even the nonsignificant results for $2 \mathrm{~Hz}: 16 \mathrm{~Hz}$ and $2 \mathrm{~Hz}: 24 \mathrm{~Hz}$ (and for $2 \mathrm{~Hz}: 32 \mathrm{~Hz}$ and $2 \mathrm{~Hz}: 40 \mathrm{~Hz}$, not shown) exhibit spatial clustering over posterior regions in the VEP time window. Couplings of other frequencies, such as $8 \mathrm{~Hz}: 16 \mathrm{~Hz}$ or $8 \mathrm{~Hz}: 40 \mathrm{~Hz}$, were not significant.

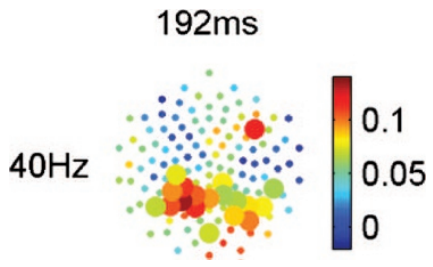

Figure 4. Fractional change in $40-\mathrm{Hz}$ local phase synchrony over a $128-\mathrm{ms}$ interval centered at $192 \mathrm{~ms}$ post-stimulus. Significant results (false discovery rate $=0.07)$ are marked with large circles.

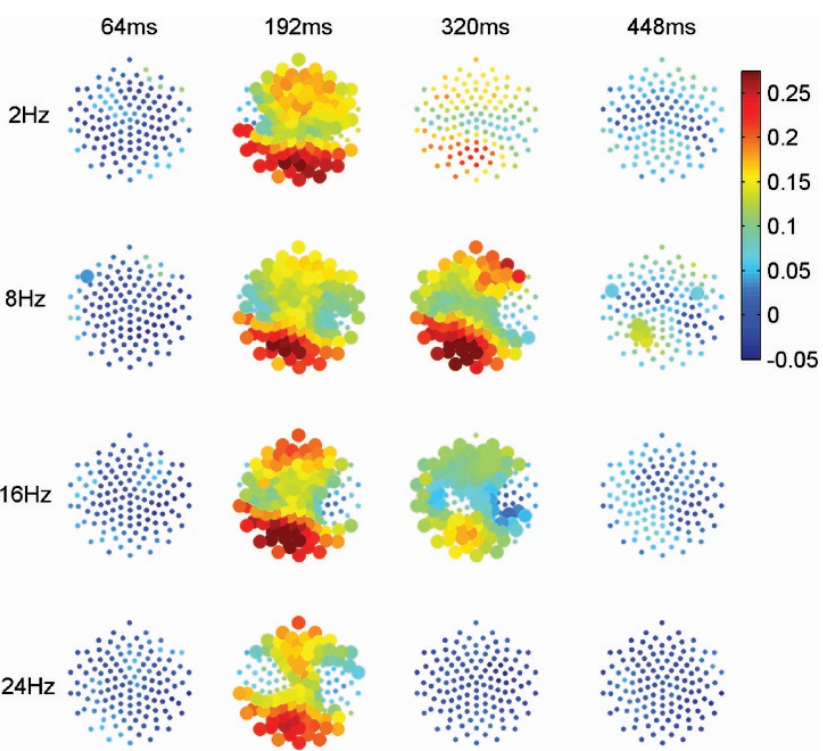

Figure 5. Phase-locking change from baseline. Significant results (false discovery rate $=0.05$ ) are shown with large circles; times are post-stimulus milliseconds. 




Figure 6. Bicoherence change from baseline. Significant results (false discovery rate $=0.05$ ) are shown with large circles; times are post-stimulus milliseconds.

\section{DISCUSSION}

Concerning traditional (averaged waveform) analysis, our results replicated previous neonatal studies. The VEP had variable temporal morphology and spatial topography across subjects and in some subjects was difficult to discern or absent $(8,27)$. The group mean VEP was not statistically significant, presumably due to the inherent variability from one infant to another in terms of amplitude, timing, morphology, and topography.

However, frequency-dependent measures did show significant changes from baseline values. As hypothesized, highfrequency $(40 \mathrm{~Hz})$ synchrony was enhanced over posterior regions around the time of the VEP (Fig. 4). Synchronization at gamma frequencies is thought to facilitate the functional performance of local networks; therefore, this result would seem to indicate that neurons in the visual cortex are responding at some time between 128 and $256 \mathrm{~ms}$ after the flash. We also hypothesized that the neural dynamics generating the VEP would include nonlinear phase coupling between frequency bands, particularly between low-frequency bands and higher frequency bands. Indeed, there was significant coupling between the $2-\mathrm{Hz}$ and $8-\mathrm{Hz}$ bands. Additionally, group means showed a similar spatial pattern for higher frequency couplings with the 2-Hz band (Fig. 6). This coupling may reflect the way in which the neural response to the flash is communicated globally through the brain, especially to more anterior regions.

The largest changes in band power occurred in posterior electrodes at $16 \mathrm{~Hz}$. The locally averaged cross-spectrum had a nearly identical spatial pattern, but with slightly greater spatial extent for the significant results (Fig. 3). The crossspectrum combines power and synchrony and is therefore ambiguous as to what neural mechanism it quantifies. Nonetheless, it performs well as a marker of VEP dynamics. Similarly, phase-locking is ambiguous regarding models of
VEP generation (additive waveform versus phase reset) but is a very robust marker of VEP dynamics, with significant responses from 2 to $32 \mathrm{~Hz}$, especially evident over posterior electrodes (Fig. 5). This frequency domain measure may prove to be more diagnostically useful than the VEP itself because phase locking exhibits highly significant results between subjects, whereas the VEP is inherently variable.

Volume conduction contributes to synchrony measurements because the potential field recorded at any given electrode is a superposition of potentials from multiple sources that affect neighboring electrodes as well. Thus, a correlation between signals from neighboring electrodes will exist even when the neural activity immediately below the electrodes is not correlated. However, we were surprised that significant changes in local and distant synchrony were not observed over a wide range of frequencies. Interestingly, the one significant result (local synchrony enhancement at $40 \mathrm{~Hz}$ ) occurred for phase synchrony but not for coherence. Whether phase synchrony or coherence is the preferred measure of synchrony remains a subject of some debate $(28,29)$.

Some of the frequency domain results showed a slight left posterior bias, consistent with the hemispheric amplitude differences seen in the VEP. Some studies have noted the occurrence of asymmetries between the two hemispheres due to idiosyncratic topography (30) and our result likely reflects this. An advantage of high-density EEG recording is that the greater number of electrodes increases the probability of successfully capturing VEP dynamics (31). Figure 3 and subsequent figures show smaller significant effects over right frontal areas that are located at nearly 180 degrees from the larger left posterior effects. Adult VEP occurs with maximum amplitude over the occiput, and some studies have reported a concomitant frontal component with opposite polarity that is not believed to be due to volume conduction from occipital sources $(32,33)$.

One limitation of frequency domain analysis is the necessary loss of temporal resolution. Unlike the VEP, the measures reported here cannot resolve neural dynamics with millisecond accuracy. Nonetheless, several of the frequency domain metrics had significant group effects during the time window of the VEP, while the group mean VEP was not significantly different from baseline. Thus, it appears that these frequency measures are more sensitive indicators of the neural dynamics of the flash response. Furthermore, the measures we studied illuminate different aspects of the underlying dynamics and provide insight into the neural mechanisms generating the local and global flash response. In future work, we will study the dependence of the flash response on sleep state in more detail. Given the robust group effects of these objective frequency domain measures in this population, we speculate that the study of a larger cohort of term and preterm infants will confirm the clinical utility of this neural dynamic assessment approach for clinical research and diagnosis.

Acknowledgments. The authors thank the reviewers for suggesting changes that improved the manuscript. 


\section{REFERENCES}

1. Taylor MJ, McCulloch DL 1992 Visual evoked potentials in infants and children. J Clin Neurophysiol 9:357-372

2. Tsuneishi S 2002 [Evaluation of the developing human visual system using flashvisual evoked potential]. No To Hattatsu 34:141-146

3. Shepherd AJ, Saunders KJ, McCulloch DL, Dutton GN 1999 Prognostic value of flash visual evoked potentials in preterm infants. Dev Med Child Neurol 41:9-15

4. Hughes JR, Rechitsky I 1996 Follow-up studies on the usefulness of the flash visual evoked potential. Clin Electroencephalogr 27:187-190

5. Iinuma K, Lombroso CT, Matsumiya Y 1997 Prognostic value of visual evoked potentials (VEP) in infants with visual inattentiveness. Electroencephalogr Clin Neurophysiol 104:165-170

6. Hartmann EE 1995 Infant visual development: an overview of studies using visual evoked potential measures from Harter to the present. Int J Neurosci 80:203-235

7. Kurtzberg D, Vaughan HG Jr 1985 Electrophysiologic assessment of auditory and visual function in the newborn. Clin Perinatol 12:277-299

8. Prager TC, Zou YL, Jensen CL, Fraley JK, Anderson RE, Heird WC 1999 Evaluation of methods for assessing visual function of infants. J AAPOS 3:275-282

9. Vanhatalo S, Kaila K 2006 Development of neonatal EEG activity: from phenomenology to physiology. Semin Fetal Neonatal Med 11:471-478

10. Jervis BW, Nichols MJ, Johnson TE, Allen E, Hudson NR 1983 A fundamental investigation of the composition of auditory evoked potentials. IEEE Trans Biomed Eng 30:43-50

11. Varela F, Lachaux JP, Rodriguez E, Martinerie J 2001 The brainweb: phase synchronization and large-scale integration. Nat Rev Neurosci 2:229-239

12. Sejnowski TJ, Paulsen O 2006 Network oscillations: emerging computational principles. J Neurosci 26:1673-1676

13. Buzsaki G, Draguhn A 2004 Neuronal oscillations in cortical networks. Science 304:1926-1929

14. Tallon-Baudry C, Bertrand O 1999 Oscillatory gamma activity in humans and its role in object representation. Trends Cogn Sci 3:151-162

15. Srinivasan R, Russell DP, Edelman GM, Tononi G 1999 Increased synchronization of neuromagnetic responses during conscious perception. J Neurosci 19:5435-5448

16. Murthy VN, Fetz EE 1992 Coherent 25- to $35-\mathrm{Hz}$ oscillations in the sensorimotor cortex of awake behaving monkeys. Proc Natl Acad Sci U S A 89:5670-5674

17. Sanes JN, Donoghue JP 1993 Oscillations in local field potentials of the primate motor cortex during voluntary movement. Proc Natl Acad Sci U S A 90:4470-4474
18. Kopell N, Ermentrout GB, Whittington MA, Traub RD 2000 Gamma rhythms and beta rhythms have different synchronization properties. Proc Natl Acad Sci U S A 97:1867-1872

19. Engel AK, Kreiter AK, Konig P, Singer W 1991 Synchronization of oscillatory neuronal responses between striate and extrastriate visual cortical areas of the cat. Proc Natl Acad Sci U S A 88:6048-6052

20. Gray CM, Konig P, Engel AK, Singer W 1989 Oscillatory responses in cat visual cortex exhibit inter-columnar synchronization which reflects global stimulus properties. Nature 338:334-337

21. von Stein A, Chiang C, Konig P 2000 Top-down processing mediated by interareal synchronization. Proc Natl Acad Sci U S A 97:14748-14753

22. Gloveli T, Dugladze T, Rotstein HG, Traub RD, Monyer H, Heinemann U, Whittington MA, Kopell NJ 2005 Orthogonal arrangement of rhythm-generating microcircuits in the hippocampus. Proc Natl Acad Sci U S A 102:13295-13300

23. Canolty RT, Edwards E, Dalal SS, Soltani M, Nagarajan SS, Kirsch HE, Berger MS, Barbaro NM, Knight RT 2006 High gamma power is phase-locked to theta oscillations in human neocortex. Science 313:1626-1628

24. Bendat JS, Piersol AG 2000 Random Data: Analysis and Measurement Procedures. Wiley, New York, p xvii

25. Nikias CL, Petropulu AP 1993 Higher-order Spectra Analysis: A Nonlinear Signal Processing Framework. PTR Prentice Hall, Englewood Cliffs, NJ, pp xxil

26. Genovese CR, Lazar NA, Nichols T 2002 Thresholding of statistical maps in functional neuroimaging using the false discovery rate. Neuroimage 15:870-878

27. Kraemer M, Abrahamsson M, Sjostrom A 1999 The neonatal development of the light flash visual evoked potential. Doc Ophthalmol 99:21-39

28. Nolte G, Bai O, Wheaton L, Mari Z, Vorbach S, Hallett M 2004 Identifying true brain interaction from EEG data using the imaginary part of coherency. Clin Neurophysiol 115:2292-2307

29. Lachaux JP, Rodriguez E, Martinerie J, Varela FJ 1999 Measuring phase synchrony in brain signals. Hum Brain Mapp 8:194-208

30. Lupton BA, Wong PK, Bencivenga R, Hill A 1990 The effect of electrode position on flash visual evoked potentials in the newborn. Doc Ophthalmol 76:73-80

31. Fifer WP, Grieve PG, Grose-Fifer J, Isler JR, Byrd D 2006 High-density electroencephalogram monitoring in the neonate. Clin Perinatol 33:679-691

32. Hobley AJ, Harding GF 1989 The topography of the P1 component of the flash visual evoked response. Doc Ophthalmol 73:119-125

33. Hughes JR, Kuruvilla A, Fino JJ 1992 Topographic analysis of visual evoked potentials from flash and pattern reversal stimuli: evidence for "travelling waves". Brain Topogr 4:215-228 